Pacific Journal of Mathematics

MIXED CUSP FORMS AND HOLOMORPHIC FORMS ON 


\title{
MIXED CUSP FORMS AND HOLOMORPHIC FORMS ON ELLIPTIC VARIETIES
}

\section{MiN Ho LEE}

\begin{abstract}
Let $E^{m}$ be an elliptic variety. We define mixed cusp forms associated to $E^{m}$ and prove that the space of mixed cusp forms coincides with the space of holomorphic $(m+1)$-forms on $E^{m}$. We also determine the geometric genus of $E^{m}$ under certain conditions.
\end{abstract}

0. Introduction. Let $\pi: E \rightarrow X$ be an elliptic fibration and $E_{0}$ the union of its regular fibers. The nonsingular variety $E^{m}$ obtained by resolving the singularities of the compactification of the fiber product

$$
\overbrace{E_{0} \times_{\pi} E_{0} \times_{\pi} \cdots \times_{\pi} E_{0}}^{m}
$$

is called an elliptic variety.

In a series of papers ([7], [8], [9]) Shokurov has constructed elliptic varieties and proved several properties of Kuga's modular varieties which are elliptic varieties of a special kind. If $m=1, E^{m}$ is simply an elliptic surface and a Kuga's modular variety is an elliptic modular surface of Shioda ([6]).

Hunt and Meyer ([3]) have introduced mixed cusp forms associated to an elliptic surface. They have shown that the space of mixed cusp forms coincides with the space of holomorphic 2-forms and used this fact to determine the geometric genus of the elliptic surface.

The main purpose of this paper is to extend the definition of mixed cusp forms of Hunt and Meyer to the case of elliptic varieties and determine the geometric genus of $E^{m}$.

1. Elliptic surfaces. Let $E$ be an elliptic surface with a global section over its base curve in the sense of Kodaira ([4]). Thus $E$ is the total space of an elliptic fibration $\pi: E \rightarrow X$ over a Riemann surface $X$ with a section $s: X \rightarrow E$ such that the generic fiber of $\pi$ is an elliptic curve.

Let $E_{0}$ be the union of the regular fibers of $\pi$ and let $X_{0}=\pi\left(E_{0}\right)$. The universal cover of $X_{0}$ is the Poincare upper half plane $\mathfrak{h}$. Let $G \subset \operatorname{PSL}(2, \mathbf{R})$ be a fuchsian group acting on $\mathfrak{h}$ by linear transformations such that $X=G \backslash \mathfrak{h}^{*}$ where $\mathfrak{h}^{*}=\mathfrak{h} \cup\{G$-cusps $\}$. Since $G=\pi_{1}\left(X_{0}\right)$, 
the natural connection on $E_{0}$ defines the monodromy representation $\chi: G \rightarrow \operatorname{SL}(2, \mathbf{R})$. If $\Gamma=\chi(G), \Gamma$ also acts on $\mathfrak{h}$ by linear transformations. Let $X_{\Gamma}=\Gamma \backslash \mathfrak{h}^{\sharp}$ where $\mathfrak{h}^{\sharp}=\mathfrak{h} \cup\{\Gamma$-cusps $\}$, and assume that the $G$-cusps and the $\Gamma$-cusps coincide. For $z \in X_{0}$, let $\Phi$ be a holomorphic 1-form on the fiber $E_{z}$ of $\pi$, and $\left\{\gamma_{1}(z), \gamma_{2}(z)\right\}$ a basis for $H_{1}\left(E_{z}, \mathbf{Z}\right)$. We define the periods $\omega_{1}$ and $\omega_{2}$ of $E$ to be the many valued holomorphic functions on $X_{0}$ given by

$$
\omega_{1}(z)=\int_{\gamma_{1}(z)} \Phi, \quad \omega_{2}(z)=\int_{\gamma_{2}(z)} \Phi .
$$

Then $\omega_{1} / \omega_{2}$ is a many valued holomorphic function from $X_{0}$ to $\mathfrak{h}$ which can be lifted to a single valued function $\bar{\omega}: \mathfrak{h} \rightarrow \mathfrak{h}$ on the universal cover $\mathfrak{h}$ of $X_{0}$. The function $\bar{\omega}$ now induces the map $\omega_{0}: G \backslash \mathfrak{h} \rightarrow$ $\Gamma \backslash \mathfrak{h}$ of the quotient spaces, and $\omega_{0}$ can be uniquely extended to the map $\omega: X \rightarrow X_{\Gamma}$ of their compactifications.

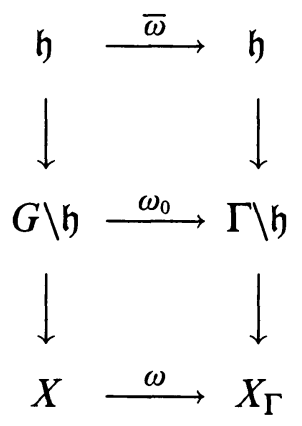

The map $\omega: X \rightarrow X_{\Gamma}$ is called the period map associated to the elliptic fibration $\pi: E \rightarrow X$.

The elliptic surface $E$ can be constructed as follows: We consider the semi-direct product $G \ltimes_{\chi}(\mathbf{Z} \times \mathbf{Z})$ given by the group law

$$
\left(g,\left(n_{1}, n_{2}\right)\right)\left(g^{\prime},\left(n_{1}^{\prime}, n_{2}^{\prime}\right)\right)=\left(g g^{\prime},\left(n_{1}, n_{2}\right) \chi\left(g^{\prime}\right)+\left(n_{1}^{\prime}, n_{2}^{\prime}\right)\right) .
$$

If $\chi(g)=\left(\begin{array}{ll}a & b \\ c & d\end{array}\right), G \ltimes_{\chi}(\mathbf{Z} \times \mathbf{Z})$ acts on $\mathfrak{h} \times \mathbf{C}$ by

$$
\left(g,\left(n_{1}, n_{2}\right)\right):(z, \zeta) \rightarrow\left(g z,(c \bar{\omega}(z)+d)^{-1}\left(\zeta+n_{1} \bar{\omega}(z)+n_{2}\right)\right)
$$

The resulting quotient

$$
\bar{E}=G \ltimes_{\chi}(\mathbf{Z} \times \mathbf{Z}) \backslash \mathfrak{h}^{*} \times \mathbf{C}
$$

has finitely many singularities, and $E$ can be recovered from $\bar{E}$ by resolving those singularities (see [4]). 
2. Elliptic varieties. Let $\pi: E \rightarrow X$ be an elliptic fibration, $\chi: G \rightarrow$ $\operatorname{SL}(2, Z)$ the monodromy representation, and $\bar{\omega}: \mathfrak{h} \rightarrow \mathfrak{h}$ the lifting of the period map. If $m$ is a positive integer, the semi-direct product $G \ltimes_{\chi}\left(\mathbf{Z}^{m} \times \mathbf{Z}^{m}\right)$ is given by the group law

$$
\left(g,\left(n_{1}, n_{2}\right)\right)\left(g^{\prime},\left(n_{1}^{\prime}, n_{2}^{\prime}\right)\right)=\left(g g^{\prime},\left(n_{1}, n_{2}\right) \chi\left(g^{\prime}\right)+\left(n_{1}^{\prime}, n_{2}^{\prime}\right)\right)
$$

where $n_{1}, n_{2}, n_{1}^{\prime}, n_{2}^{\prime} \in R^{m}$ are column vectors. If $\chi(g)=\left(\begin{array}{ll}a & b \\ c & d\end{array}\right)$, the group $G \ltimes_{\chi}\left(\mathbf{Z}^{m} \times \mathbf{Z}^{m}\right)$ acts on $\mathfrak{h} \times \mathbf{C}^{m}$ properly discontinuously by

$$
\left(g,\left(n_{1}, n_{2}\right)\right):(z, \zeta) \rightarrow\left(g z,(c \bar{\omega}(z)+d)^{-1}\left(\zeta+n_{1} \bar{\omega}(z)+n_{2}\right)\right)
$$

where $n_{1}, n_{2} \in R^{m}$ and $\zeta \in \mathbf{C}^{m}$ are column vectors. Let $\bar{E}^{m}$ be the compactification of the analytic variety

$$
G \ltimes_{\chi}\left(\mathbf{Z}^{m} \times \mathbf{Z}^{m}\right) \backslash \mathfrak{h} \times \mathbf{C}^{m} .
$$

Let $E^{m}$ be the variety obtained from $\bar{E}^{m}$ by resolving the singularities (see [7] and [8] for details). The map $\pi: E \rightarrow X$ naturally induces the map $\pi^{m}: E^{m} \rightarrow X$ which is a fiber bundle whose generic fiber is the product of $m$ elliptic curves. The fiber variety $E^{m}$ is called the elliptic variety associated to the elliptic fibration $\pi: E \rightarrow X$.

3. Mixed cusp forms. We shall extend the definition of mixed cusp forms given in [3] to the case of $m \geq 1$. Let $G \in \operatorname{PSL}(2, \mathbf{R})$ be a fuchsian group of the first kind acting on $\mathfrak{h}$ by linear transformations and $\chi: G \rightarrow \operatorname{SL}(2, Z)$ a homomorphism such that $\Gamma=\chi(G)$ is of finite index in $\operatorname{SL}(2, Z)$. Then $\Gamma$ also acts on $\mathfrak{h}$ by linear transformations. We assume that the inverse images of parabolic subgroups of $\Gamma$ under $\chi$ are parabolic subgroups of $G$. Let $\bar{\omega}: \mathfrak{h} \rightarrow \mathfrak{h}$ be a holomorphic map such that

$$
\bar{\omega}(g z)=\chi(g) \bar{\omega}(z)
$$

for all $g \in G$ and $z \in \mathfrak{h}$.

Definition 3.1. Let $m$ be a positive integer. A holomorphic function $f: \mathfrak{h} \rightarrow \mathbf{C}$ is a mixed cusp form of type $(2, m)$ if it satisfies the following conditions:

(i) $f(g z)=(c z+d)^{2}\left(c_{1} \bar{\omega}(z)+d_{1}\right)^{m} f(z)$ if $g=\left(\begin{array}{ll}a & b \\ c & d\end{array}\right) \in G$ and $\chi(g)=\left(\begin{array}{ll}a_{1} & b_{1} \\ c_{1} & d_{1}\end{array}\right) \in \Gamma$;

(ii) $f$ vanishes at the $G$-cusps.

The space of all mixed cusp forms of type $(2, m)$ associated to $G$, $\bar{\omega}$, and $\chi$ is denoted by $S_{2, m}(G, \bar{\omega}, \chi)$. 
THEOREM 3.2. Let $E^{m}$ be the elliptic variety associated to the elliptic fibration $\pi: E \rightarrow X$ with $X=G \backslash \mathfrak{h}^{*}$; let $\chi: G \rightarrow \operatorname{SL}(2, \mathbf{Z})$ be the monodromy representation with $\Gamma=\chi(G)$, and $\bar{\omega}: \mathfrak{h} \rightarrow \mathfrak{h}$ the lifting of the period map $\omega: X \rightarrow X_{\Gamma}$. Then we have

$$
H^{0}\left(E^{m}, \Omega^{m+1}\right)=S_{2, m}(G, \bar{\omega}, \chi)
$$

where $\Omega^{m+1}$ is the sheaf of holomorphic $(m+1)$-forms.

Proof. Let $X_{0}$ be the union of the regular fibers of $\pi$. Then as described in $\S 3$,

$$
\left.E^{m}\right|_{X_{0}}=G \ltimes_{\chi}\left(\mathbf{Z}^{m} \times \mathbf{Z}^{m}\right) \backslash \mathfrak{h} \times \mathbf{C}^{m}
$$

where, if $\chi(g)=\left(\begin{array}{ll}a_{1} & b_{1} \\ c_{1} & d_{1}\end{array}\right), G \ltimes_{\chi}\left(\mathbf{Z}^{m} \times \mathbf{Z}^{m}\right)$ acts on $\mathfrak{h} \times \mathbf{C}^{m}$ by

$$
\left(g,\left(n_{1}, n_{2}\right)\right):(z, \zeta) \rightarrow\left(g z,\left(c_{1} \bar{\omega}(z)+d_{1}\right)^{-1}\left(\zeta+n_{1} \bar{\omega}(z)+n_{2}\right)\right) .
$$

Thus holomorphic $(m+1)$-forms on $\left.E^{m}\right|_{X_{0}}$ are simply holomorphic $(m+1)$-forms on $\mathfrak{h} \times \mathbf{C}^{m}$ invariant under $G \ltimes_{\chi}\left(\mathbf{Z}^{m} \times \mathbf{Z}^{m}\right)$. A holomorphic $(m+1)$-form on $\mathfrak{h} \times \mathbf{C}^{m}$ is of the form

$$
\Theta=F\left(z, \zeta_{1}, \ldots, \zeta_{m}\right) d z \wedge d \zeta_{1} \wedge d \zeta_{2} \wedge \cdots \wedge d \zeta_{m}
$$

where $F$ is holomorphic. Given a fixed point $x \in X_{0}, \Theta$ descends to an $m$-form on the fiber $E_{x}^{m}$ of $\pi^{m}: E^{m} \rightarrow X$. Since $E_{x}^{m}$ is a product of $m$ elliptic curves, it follows that

$$
\operatorname{dim} H^{0}\left(E_{x}^{m}, \Omega^{m}\right)=1 \text {. }
$$

Therefore $\left.F\right|_{E_{x}^{m}}$ is constant, and $F\left(z, \zeta_{1}, \ldots, \zeta_{m}\right)$ depends only on $z$, say

$$
F\left(z, \zeta_{1}, \ldots, \zeta_{m}\right)=f(z)
$$

Since $\Theta$ is invariant under the group action, if $g=\left(\begin{array}{ll}a & b \\ c & d\end{array}\right)$, we have

$$
\begin{aligned}
\Theta= & f(z) d z \wedge d \zeta_{1} \wedge \cdots \wedge d \zeta_{m} \\
= & f(g z) d(g z) \wedge d\left(\left(c_{1} \bar{\omega}(z)+d_{1}\right)^{-1}\left(\zeta_{1}+\bar{\omega}(z) n_{11}+n_{21}\right)\right) \\
& \wedge \cdots \wedge d\left(\left(c_{1} \bar{\omega}(z)+d_{1}\right)^{-1}\left(\zeta_{m}+\bar{\omega}(z) n_{1 m}+n_{2 m}\right)\right) \\
= & f(g z)(c z+d)^{-2}\left(c_{1} \bar{\omega}(z)+d_{1}\right)^{-m} d z \wedge d \zeta_{1} \wedge d \zeta_{2} \wedge \cdots \wedge d \zeta_{m}
\end{aligned}
$$

Thus it follows that

$$
f(g z)=(c z+d)^{2}\left(c_{1} \bar{\omega}(z)+d_{1}\right)^{m} f(z)
$$

which is the condition (i) for $S_{2, m}(G, \bar{\omega}, \chi)$. 
Now we shall show that $\Theta$ can be extended to $E^{m}$ if and only if $f$ satisfies the condition (ii) for $S_{2, m}(G, \bar{\omega}, \chi)$. By Theorem 3.1 in [5] $\Theta$ can be extended to $E^{m}$ if and only if

$$
\int_{E^{m} \mid x_{0}} \Theta \wedge \bar{\Theta}<\infty
$$

But we have

$$
\int_{\left.E^{m}\right|_{x_{0}}} \Theta \wedge \bar{\Theta}=\int_{W} \Theta \wedge \bar{\Theta}
$$

where $W$ is a fundamental domain in $\mathfrak{h} \times \mathbf{C}^{m}$ with respect to the group $G \ltimes_{\chi}\left(\mathbf{Z}^{m} \times \mathbf{Z}^{m}\right)$. If $U$ is a fundamental domain in $\mathfrak{h}$ with respect to $G$ and $U_{1}$ is a lifting of $U$ to $W$, then $W$ can be chosen such that

$$
W=\left\{(z, \zeta) \mid z \in U_{1}, \zeta_{k}=t_{1, k}+t_{2, k} z ; 0 \leq t_{j, k} \leq 1\right\} .
$$

Then we have

$$
\begin{aligned}
\int_{W} \Theta \wedge \bar{\Theta} & =\int_{W}|f(z)|^{2} d z \wedge\left(\bigwedge_{k=1}^{m} d \zeta_{k}\right) \wedge d \bar{z} \wedge\left(\bigwedge_{k=1}^{m} d \bar{\zeta}_{k}\right) \\
& =C \int_{U}|f(z)|^{2}(\operatorname{Im}(z))^{m} d z \wedge d \bar{z}
\end{aligned}
$$

where $C$ is some nonzero complex number. Since $\Theta$ is analytic on $\mathfrak{h}$ and the $G$-cusps are isolated, it follows easily that

$$
\int_{U}|f(z)|^{2}(\operatorname{Im}(z))^{m} d z \wedge d \bar{z}<\infty
$$

if and only if $f$ vanishes at the $G$-cusps.

4. Line bundles associated to forms. In this section we shall construct the line bundles associated to cusp forms as in [1]. Let $G \subset$ $\operatorname{PSL}(2, \mathbf{R})$ and let $\mathscr{O}_{\mathfrak{h}}$ be the sheaf of germs of holomorphic functions on $\mathfrak{h}$. If $\mathfrak{h}^{*}=\mathfrak{h} \cup\{G$-cusps $\}, \mathscr{O}_{\mathfrak{h}}$ can be extended to the sheaf $\mathscr{O}_{\mathfrak{h}^{*}}$ on $\mathfrak{h}^{*}$ as follows: Let $i: \mathfrak{h} \rightarrow \mathfrak{h}^{*}$ be the inclusion map and $s$ a $G$-cusp such that $s=g(\infty), g \in G$. We define the section $\mathscr{O}_{\mathfrak{h}^{*}, s}$ of $\mathscr{O}_{\mathfrak{h}^{*}}$ over $s$ to be the set of all elements in the direct image sheaf $i_{*} \mathscr{O}_{\mathfrak{h}}$ which are meromorphic there, i.e.

$$
\mathscr{O}_{\mathfrak{h}^{*}, s}=\left\{f \in i_{*} \mathscr{O}_{\mathfrak{h}} \mid \lim _{z \rightarrow \infty}\left(f(g z) / z^{m}\right) \text { is finite for some } m \in \mathbf{Z}\right\} .
$$

$G$ acts on $\mathscr{O}_{\mathfrak{h}}$ by $\gamma f(z)=f\left(\gamma^{-1} z\right)$, and it also acts on the sheaf $\mathscr{F}=$ $\mathscr{O}_{\mathfrak{h} *} \otimes_{\mathbf{C}} \mathbf{C}^{2}$ by

$$
g\left(\begin{array}{l}
f_{1} \\
f_{2}
\end{array}\right)(z)=\left(\begin{array}{ll}
a & b \\
c & d
\end{array}\right)\left(\begin{array}{l}
f_{1} \\
f_{2}
\end{array}\right)\left(g^{-1} z\right)
$$


where $g=\left(\begin{array}{ll}a & b \\ c & d\end{array}\right) \in G$. Let $\mathscr{F}^{1}$ be the subsheaf of $\mathscr{F}$ generated by the global section $\left(\begin{array}{l}z \\ 1\end{array}\right)$, i.e.

$$
\mathscr{F}^{1}=\left\{\left(\begin{array}{c}
z \\
1
\end{array}\right) f \mid f \in \mathscr{O}_{\mathfrak{h}^{*}}\right\} \subset \mathscr{F} .
$$

LEMMA 4.1. $\mathscr{F}^{1}$ is $G$-invariant.

Proof. For each $g=\left(\begin{array}{ll}a & b \\ c & d\end{array}\right) \in G$, we have

$$
\begin{aligned}
g\left(\begin{array}{l}
z \\
1
\end{array}\right) & =\left(\begin{array}{ll}
a & b \\
c & d
\end{array}\right)\left(\begin{array}{c}
g^{-1} z \\
1
\end{array}\right)=\left(\begin{array}{ll}
a & b \\
c & d
\end{array}\right)\left(\begin{array}{c}
(d z-b) /(-c z+a) \\
1
\end{array}\right) \\
& =(-c z+a)^{-1}\left(\begin{array}{l}
z \\
1
\end{array}\right) \in \mathscr{F}^{1}
\end{aligned}
$$

which proves the lemma.

Let $\mathscr{F}_{G}^{1}$ be the sheaf on $X_{G}=G \backslash \mathfrak{h}^{*}$ descended from $\mathscr{F}^{1}$, and let

$$
\mathscr{F}^{k}=\left(\mathscr{F}^{1}\right)^{\otimes k}, \quad \mathscr{F}_{G}^{k}=\left(\mathscr{F}_{G}^{1}\right)^{\otimes k} .
$$

Proposition 4.2. If $A_{k}(G)$ denotes the space of holomorphic automorphic forms of weight $k$ on $\mathfrak{h}$, then

$$
A_{k}(G)=\Gamma\left(X_{G}, \mathscr{F}_{G}^{k}\right), \quad k \geq 1 .
$$

Proof. This follows easily from the way $G$ acts on $\mathscr{F}$ (see [1, Theorem 1.4] for details).

Let $\Sigma \subset \mathfrak{h}^{*}$ be the set of $G$-cusps. Then the space of cusp forms of weight $k S_{k}(G)$ is by Proposition 4.2 the space of global sections of

$$
\mathscr{F}_{G_{0}}^{k}:=\left(\mathscr{F}^{k} \otimes \mathscr{O}_{\mathfrak{h}^{*}}(-\Sigma)\right)_{G}
$$

where $(\cdot)_{G}$ denotes the fixed sheaf (cf. $\left.[1, \S 0]\right)$, and $\mathscr{O}_{\mathfrak{h}^{*}}(-\Sigma)$ is the sheaf of functions on $\mathfrak{h}^{*}$ which are holomorphic on $\mathfrak{h}$ and zero at the $G$-cusps.

Proposition 4.3. If $G$ has no elements of finite order, then

$$
S_{k+2}(G)=\Gamma\left(X_{G}, \mathscr{F}_{G}^{k} \otimes \Omega_{X_{G}}^{1}\right)
$$

where $\Omega_{X_{G}}^{1}$ is the sheaf of holomorphic 1-forms on $X_{G}$.

Proof. By Proposition 1.3 in [1] the identification $\mathscr{F}^{2}=\Omega_{\mathfrak{h}^{*}}^{1}$ induces

$$
\mathscr{F}_{G}^{2}=\Omega_{X_{G}}^{1}(\Sigma)
$$


where $\Omega_{X_{G}}^{1}(\Sigma)$ is the space of meromorphic 1-forms on $X_{G}$ which are holomorphic on $X_{G} \backslash\{G$-cusps $\}$ and have poles of order at most one at the $G$-cusps. Since $G$ has no elements of finite order, the sheaf $\mathscr{F}^{k}$ on $\mathfrak{h}^{*}$ is stalkwise free therefore by Proposition 0.3 in [1] $\mathscr{F}_{G_{0}}^{k}$ descends to the quotient $X_{G}=G \backslash \mathfrak{h}^{*}$. Hence we have

$$
\begin{aligned}
\mathscr{F}_{G_{0}}^{k+2} & =\left(\mathscr{F}^{k} \otimes \mathscr{F}^{2} \otimes \mathscr{O}_{\mathfrak{h}^{*}}(-\Sigma)\right)_{G} \\
& =\mathscr{F}_{G}^{k} \otimes\left(\Omega_{X_{G}}^{1}(\Sigma) \otimes \mathscr{O}_{X_{G}}(-\Sigma)\right)=\mathscr{F}_{G}^{k} \otimes \Omega_{X_{G}}^{1} .
\end{aligned}
$$

Therefore we get

$$
S_{k+2}(G)=\Gamma\left(X_{G}, \mathscr{F}_{G_{0}}^{k+2}\right)=\Gamma\left(X_{G}, \mathscr{F}_{G}^{k} \otimes \Omega_{X_{G}}^{1}\right) .
$$

5. The geometric genus of $E^{m}$. Let $E^{m}$ be the elliptic variety associated to an elliptic fibration $\pi: E \rightarrow X$. Let $E_{0}$ be the union of regular fibers of $\pi$. If $X_{0}=\pi\left(E_{0}\right)$ and $G=\pi_{1}\left(X_{0}, *\right) \subset \operatorname{PSL}(2, \mathbf{R})$, then

$$
X_{0}=G \backslash \mathfrak{h}, \quad X=G \backslash \mathfrak{h}^{*}
$$

where $\mathfrak{h}^{*}=\mathfrak{h} \cup\{G$-cusps $\}$. Let $\chi: G \rightarrow \operatorname{SL}(2, Z)$ be the monodromy representation with $\Gamma=\chi(G)$. If $\omega^{*}\left(\mathscr{F}_{\Gamma_{0}}^{m}\right)$ is the sheaf over $X$ obtained by pulling back $\mathscr{F}_{\Gamma_{0}}^{m}$ via the period map $\omega: X \rightarrow X_{\Gamma}$, we define the line bundle $\mathscr{F}_{\omega}$ by

$$
\mathscr{F}_{\omega}^{m}:=\mathscr{F}_{G_{0}}^{2} \otimes \omega^{*}\left(\mathscr{F}_{\Gamma_{0}}^{m}\right)=\Omega_{X}^{1} \otimes \omega^{*}\left(\mathscr{F}_{\Gamma_{0}}^{m}\right):
$$

Proposition 5.1. If $G$ has no elements of finite order, then

$$
S_{2, m}(G, \bar{\omega}, \chi)=H^{0}\left(X, \mathscr{F}_{\omega}^{m}\right) .
$$

Proof. Since $G$ has no elements of finite order, $\omega$ maps $G$-cusps into $\Gamma$-cusps. Thus the proposition follows from the fact that the sections of $\mathscr{F}_{\omega}^{m}$ transform in exactly the same way as the mixed cusp forms do in $S_{2, m}(G, \bar{\omega}, \chi)$.

If $p_{g}\left(E^{m}\right)$ denotes the geometric genus of $E^{m}$, then by Theorem 3.2 and Proposition 5.1 we have

$$
\begin{aligned}
p_{g}\left(E^{m}\right) & =\operatorname{dim} H^{0}\left(E^{m}, \Omega^{m+1}\right) \\
& =\operatorname{dim} S_{2, m}(G, \bar{\omega}, \chi)=\operatorname{dim} H^{0}\left(X, \mathscr{F}_{\omega}^{m}\right) .
\end{aligned}
$$

THEOREM 5.2. Let $E^{m}$ be the elliptic variety associated to the elliptic fibration $\pi: E \rightarrow X$ with $X=G \backslash \mathfrak{h}^{*}$. If $G$ contains no elements of finite 
order and if the period map $\omega: X \rightarrow X_{\Gamma}$ sends $G$-cusps to $\Gamma$-cusps, then the geometric genus of $E^{m}$ is given by

$$
p_{g}\left(E^{m}\right)=(m+1)(g(X)-1)-(m / 2) \Sigma\left(e_{z}-1\right)+N m / 2
$$

where $e_{z}$ is the ramification index of $\omega$ at $z \in X, N$ is the number of $G$-cusps, and $g(X)$ is the genus of $X$.

Proof. Since $\operatorname{deg}\left(\omega^{*} \mathscr{F}_{\Gamma_{0}}^{m}\right)>0, \operatorname{deg} \mathscr{F}_{\omega}^{m}>2 g-2$. Thus $\mathscr{F}_{\omega}^{m}$ is nonspecial (see e.g. [2, p. 296]), and hence by the Riemann-Roch theorem we have

$$
\begin{aligned}
\operatorname{dim} H^{0}\left(X, \mathscr{F}_{\omega}^{m}\right) & =1-g(X)+\operatorname{deg}\left(\mathscr{F}_{\omega}^{m}\right) \\
& =1-g(X)+\operatorname{deg} \Omega_{X}^{1}+(\operatorname{deg} \omega)\left(\operatorname{deg} \mathscr{F}_{\Gamma_{0}}^{m}\right) \\
& =1-g(X)+2 g(X)-2+m(\operatorname{deg} \omega)\left(\operatorname{deg} \mathscr{F}_{\Gamma_{0}}^{1}\right) \\
& =g(X)-1+m(\operatorname{deg} \omega)\left(2 g\left(X_{\Gamma}\right)-2+t\right) / 2
\end{aligned}
$$

where $t$ is the number of $\Gamma$-cusps. Now by Hurwitz's theorem

$$
2 g(X)-2=(\operatorname{deg} \omega)\left(2 g\left(X_{\Gamma}\right)-2\right)+\sum_{z \in X}\left(e_{z}-1\right) \text {. }
$$

So we have

$\operatorname{dim} H^{0}\left(X, \mathscr{F}_{\omega}^{m}\right)=(m+1)(g(X)-1)-(m / 2) \Sigma\left(e_{z}-1\right)+m t(\operatorname{deg} \omega) / 2$.

Now the theorem follows from the fact that $(\operatorname{deg} \omega) t=N$.

\section{REFERENCES}

[1] P. Bayer and J. Neukirch, On automorphic forms and Hodge theory, Math. Ann., 257 (1981), 135-155.

[2] R. Hartshorne, Algebraic Geometry, Springer-Verlag, 1977.

[3] B. Hunt and W. Meyer, Mixed automorphic forms and invariants of elliptic surfaces, Math. Ann., 271 (1985), 53-80.

[4] K. Kodaira, On compact analytic surfaces, II-III, Ann. Math., 77 (1963), 563626; 78 (1963), 1-40.

[5] H. B. Laufer, On rational singularities, Amer. J. Math., 94 (1972), 597-608.

[6] T. Shioda, On elliptic modular surfaces, J. Math. Soc. Japan, 24 (1972), 20-59.

[7] V. V. Shokurov, Holomorphic differential forms of higher degree on Kuga's modular varieties, Math. USSR Sb., 30 (1976), 119-142.

[8] _ The study of the homology of Kuga varieties, Math. USSR Izv., 16 (1981), 399-418.

[9] _ Shimura integrals of cusp forms, Math. USSR Izv., 16 (1981), 603-646.

Received February 20, 1987. 


\title{
PACIFIC JOURNAL OF MATHEMATICS EDITORS
}

\author{
V. S. VARADARAJAN \\ (Managing Editor) \\ University of California \\ Los Angeles, CA 90024 \\ HERBERT Clemens \\ University of Utah \\ Salt Lake City, UT 84112 \\ R. FINN \\ Stanford University \\ Stanford, CA 94305
}

\author{
HERMANN FLASCHKA \\ University of Arizona \\ Tucson, AZ 85721
}

Ramesh A. Gangolli University of Washington Seattle, WA 98195

VAUGHAN F. R. JONES University of California Berkeley, CA 94720
ROBION KIRBY

University of California

Berkeley, CA 94720

C. C. MOORE

University of California

Berkeley, CA 94720

HAROLD STARK

University of California, San Diego La Jolla, CA 92093

\section{ASSOCIATE EDITORS}

\author{
R. ARENS \\ E. F. BECKENBACH \\ B. H. NEUMANN \\ F. WOLF \\ K. YOSHIDA \\ (1906-1982)

\section{SUPPORTING INSTITUTIONS}

\section{UNIVERSITY OF ARIZONA} \\ UNIVERSITY OF BRITISH COLUMBIA \\ CALIFORNIA INSTITUTE OF TECHNOLOGY \\ UNIVERSITY OF CALIFORNIA \\ MONTANA STATE UNIVERSITY \\ UNIVERSITY OF NEVADA, RENO \\ NEW MEXICO STATE UNIVERSITY \\ OREGON STATE UNIVERSITY \\ UNIVERSITY OF OREGON \\ UNIVERSITY OF SOUTHERN CALIFORNIA \\ STANFORD UNIVERSITY \\ UNIVERSITY OF HAWAII \\ UNIVERSITY OF TOKYO \\ UNIVERSITY OF UTAH \\ WASHINGTON STATE UNIVERSITY \\ UNIVERSITY OF WASHINGTON
}

The Supporting Institutions listed above contribute to the cost of publication of this Journal, but they are not owners or publishers and have no responsibility for its content or policies.

Mathematical papers intended for publication in the Pacific Journal of Mathematics should be in typed form or offset-reproduced (not dittoed), double spaced with large margins. Please do not use built up fractions in the text of the manuscript. However, you may use them in the displayed equations. Underline Greek letters in red, German in green, and script in blue. The first paragraph must be capable of being used separately as a synopsis of the entire paper. In particular it should contain no bibliographic references. Please propose a heading for the odd numbered pages of less than 35 characters. Manuscripts, in triplicate, may be sent to any one of the editors. Please classify according to the scheme of Math. Reviews, Index to Vol. 39. Supply name and address of author to whom proofs should be sent. All other communications should be addressed to the managing editor, or Elaine Barth, University of California, Los Angeles, California 90024

There are page-charges associated with articles appearing in the Pacific Journal of Mathematics. These charges are expected to be paid by the author's University, Government Agency or Company. If the author or authors do not have access to such Institutional support these charges are waived. Single authors will receive 50 free reprints; joint authors will receive a total of 100 free reprints. Additional copies may be obtained at cost in multiples of 50 .

The Pacific Journal of Mathematics is issued monthly as of January 1966. Regular subscription rate: $\$ 190.00$ a year (5 Vols., 10 issues). Special rate: $\$ 95.00$ a year to individual members of supporting institutions.

Subscriptions, orders for numbers issued in the last three calendar years, and changes of address should be sent to Pacific Journal of Mathematics, P.O. Box 969, Carmel Valley, CA 93924, U.S.A. Old back numbers obtainable from Kraus Periodicals Co., Route 100, Millwood, NY 10546.

The Pacific Journal of Mathematics at P.O. Box 969, Carmel Valley, CA 93924 (ISSN 0030-8730) publishes 5 volumes per year. Application to mail at Second-class postage rates is pending at Carmel Valley, California, and additional mailing offices. Postmaster: send address changes to Pacific Journal of Mathematics, P.O. Box 969, Carmel Valley, CA 93924.

PUBLISHED BY PACIFIC JOURNAL OF MATHEMATICS, A NON-PROFIT CORPORATION Copyright (C) 1988 by Pacific Journal of Mathematics 


\section{Pacific Journal of Mathematics}

\section{Vol. 132, No. $2 \quad$ February, 1988}

Jeffery Marc Bergen and Luisa Carini, A note on derivations with power central values on a Lie ideal ..............................209

Alfonso Castro and Sumalee Unsurangsie, A semilinear wave equation

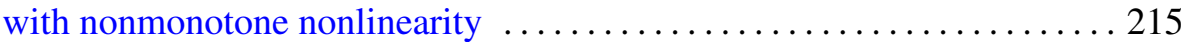

Marius Dadarlat, On homomorphisms of matrix algebras of continuous

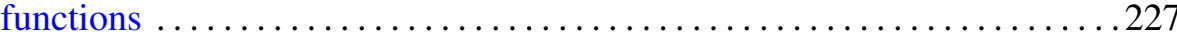

A. Didierjean, Quelques classes de cobordisme non orienté refusant de se

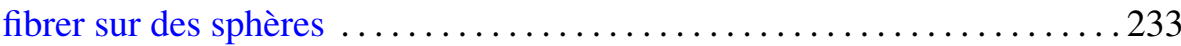

Edward George Effros and Zhong-Jin Ruan, On matricially normed spaces

Sherif El-Helaly and Taqdir Husain, Orthogonal bases are Schauder bases

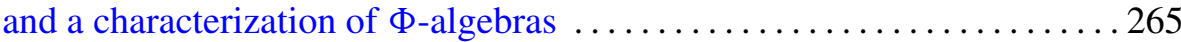

Edward Richard Fadell and Peter N-S Wong, On deforming $G$-maps to be

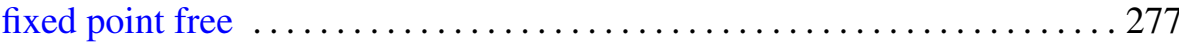

Jean-Jacques Gervais, Stability of unfoldings in the context of equivariant

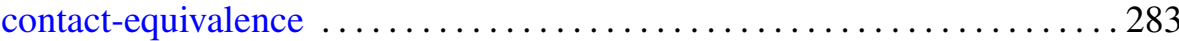

Douglas Martin Grenier, Fundamental domains for the general linear group

Ronald Scott Irving and Brad Shelton, Loewy series and simple projective

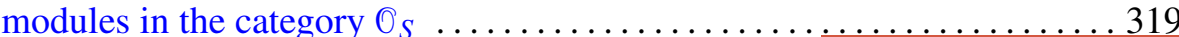

Russell Allan Johnson, On the Sato-Segal-Wilson solutions of the K-dV equation

Thomas Alan Keagy and William F. Ford, Acceleration by subsequence transformations

Min Ho Lee, Mixed cusp forms and holomorphic forms on elliptic varieties

Charles Livingston, Indecomposable surfaces in 4-space 